\title{
Evaluation the Correlation between Ki67 and 5 Years Disease Free Survival of Breast Cancer Patients
}

\author{
S.M. Hosseini' ${ }^{1}$ H. Shahbaziyan ${ }^{1}$, S. Razmjoo ${ }^{1}$ and N. Jasemizade $^{2}$ \\ ${ }^{1}$ Department of Radiation and Oncology of Golestan Hospital, \\ Ahvaz Jundishapur University of Medical Sciences, Ahvaz, Iran . \\ ${ }^{2} \mathrm{MD}$ student, Faculty of Medicine, Ahvaz Jundishapur \\ University of Medical Sciences, Ahvaz, Iran.
}

DOI: http://dx.doi.org/10.13005/bbra/1894

(Received: 30 August 2015; accepted: 09 October 2015)

\begin{abstract}
Breast cancer is the most frequently diagnosed life-threatening cancer among women worldwide. Ki67 as a nuclear proliferation marker is used as a predictive marker for response to chemotherapy. In this study we retrospectively evaluated the association between Ki67 and 5 years disease free survival (5yDFS) in breast cancer patients. In addition, the correlation between ER, PR, HER2, and P53 and 5yDFS was studied. Total of 365 patients with breast cancer previously treated with surgery and/or chemoradiotherapy during 2001 to 2008 in department of were included in the study. All patients had invasive ductal carcinoma in the pathology. Age, Ki67, ER, PR, HER2, and P53 were studied. Her2/Neu expression level was assessed by reaction with antibodies against HER2/neu. Proliferation level was determined by reaction with antibodies against Ki-67: the level e" $14 \%$ considered high and the level $<14 \%$ considered low. The frequency and percentage were used for qualification data and mean and Standard Deviation (SD) for the quantitative data. The mean level of ki67 was $16.85 \pm 16.63$ and the frequency of high ki67 was $203(55.6 \%)$. The mean age of breast cancer patients was $47.2 \pm 10.53$. ER status was positive in 242 patients $(\mathbf{6 6 . 3} \%)$. One hundred and fifty four breast cancer patients had PR negative (42.2\%). The HER2 overexpression was identified in 96 tumors $(26.3 \%)$. The P53 status in tumors was positive in $166(45.5 \%)$. The $55.6 \%$ of the patients had $5 y$ DFS. The $67.5 \%$ and $40.7 \%$ of breast cancer patients respectively with low level of Ki67 and high level of Ki67 showed significant correlation with 5yDFS. Our findings showed increased level of 5yDFS in ER positive breast cancer patients. In addition, the chance of high Ki67 is increased in the ER negative, HER2 negative, and P53 positive breast cancer patients.
\end{abstract}

Key words: Breast Cancer, molecular marker, Estrogen Receptor, Progesterone Receptor, HER2, Ki67, 5yDFS, P53.

Breast cancer is the most frequently diagnosed life-threatening cancer among women worldwide. In less-developed countries, it is the leading cause of cancer death in women; in developed countries, however, it has been surpassed by lung cancer death in women ${ }^{1}$. In the

\footnotetext{
* To whom all correspondence should be addressed. Tel.: 09163135583; Fax: 06133743057

E-mail: kiahossainy@yahoo.com
}

United States, approximately 231,840 new cases of female invasive breast cancer are predicted to occur in 2015, along with 2350 cases in men². The prevalence of breast cancer is about one third of all cancers among women. The incidence of the disease among Iranian women is increasing ${ }^{3}$. Uncontrolled proliferation is an important feature of malignant tumors. Although treatment of metastatic breast cancer is difficult ${ }^{4}$, there are improvements in increasing survival of these patients $^{5-7}$. The nuclear proliferation marker Ki67 
is used as a predictive marker for response to chemotherapy and is associated with patient prognosis in breast cancer ${ }^{8}$. Such clinical characteristics as age, menstrual status, tumor size, lymph node status and morphological characteristics of the tumor (histological type, grade, and lymphatic/vascular invasion) are traditionally the most important prognostic factors. However, during the last decades understanding of tumor nature has been greatly improved by molecular biology studies that have allowed application of tumor's molecular features for prognosis of the disease course. Most of molecular markers that have been studied so far, demonstrate the ability of cells to malignant growth [9]. Estrogen and progesterone receptors (ER, PR) were the first predictive molecular markers for breast cancer. Patients with positive status of these steroid hormone receptors generally have high sensitivity to hormone therapy. Next marker that was included in clinical practice is human epidermal receptor 2 (HER2/Neu), whose positive status in patients with breast cancer correlates with high sensitivity to targeted therapy with Trastuzumab [10]. Determining molecular markers of breast cancer in combination with recent technique in early diagnosis as well as alternative treatment of breast cancer can significantly enhance the management of breast cancer ${ }^{11,12,13-15}$.

In this study we retrospectively evaluated the association between Ki67 and 5 years disease free survival (5yDFS) in breast cancer patients. In addition, we evaluated the correlation between ER, PR, HER2, and P53 with 5yDFS.

\section{MATERIALSAND METHODS}

\section{Participants}

Three hundred and sixty five patients with breast cancer previously treated with surgery and/ or chemo-radiotherapy in the Radiotherapy department of Ahvaz Golestan Hospital (Iran) during 2001 to 2008 were included in the study. All of them had invasive ductal carcinoma in the pathology. The recording parameters were: age, Ki67 (percent), ER, PR, HER2, and P53. Some files were followed up for 5 years after the time of pathology but we followed up patient with incomplete follow up by phone number. Excluded files were the files without pathological index, the patients that were not margin free in pathology or were metastatic at the time of diagnosis.

\section{Parameters}

Her2/Neu expression level was assessed by reaction with antibodies against HER2/neu. For interpretation of the reaction the following criteria were recorded: 0 (negative): no staining is observed or membranous staining is observed in less than $10 \%$ of the tumor cells. 1+ (negative): A faint/barely perceptible staining is detected in more than $10 \%$ of the tumor cells. The cells are stained in part of their membrane. $2+$ (equivocal): A weak to moderate complete membrane staining is observed in more than $10 \%$ of the tumor cells. $3+$ (positive): A strong complete membrane staining is observed in more than $30 \%$ of the tumor cells. Tumors with expression level 0 and $1+$ and 2+ considered HER2/Neunegative, and with $3+$ considered HER2 positive. Proliferation level was determined by reaction with antibodies against Ki-67: the level e"14\% considered high and the level $<14 \%$ considered low.

P53-status of tumors was determined by the level of accumulation in the nuclei of tumor cells. We recorded this parameter as positive or negative from the medical recordings. ER and PR that recorded from the histology of cancer in the files were considered positive and negative. The presence of 5yDFS for the patients who did not have metastasis or recurrence considered positive (Yes). In addition, for the patients who had metastasis or recurrence during 5 years following the time of pathology was considered negative (No).

\section{Data analysis}

The descriptive statistics were used to analyze the data. The frequency and percentage were used for qualification data and mean and Standard Deviation (SD) for the quantitative data. All data were analyzed with independent T-Test, Mann Whitney, Analysis of variance (ANOVA) and General Linear Model (GLM) tests using statistical package of SPSS (version 20).

\section{RESULTS AND DISCUSSION}

The mean level of ki67 was $16.85 \pm 16.63$ and the frequency of high ki67 was 203 (55.6\%). We compared it in the frequency contribution that did not show significant differences so we entered 
ki67_Cat in our evaluations. Of total, 162 breast cancer patients had low ki67 (44.4\%). The mean age of breast cancer patients was $47.2 \pm 10.53$. ER status was positive in 242 patients (66.3\%) and negative in 123 patients (33.7\%). One hundred and fifty four breast cancer patients had PR negative (42.2\%) and 211 had PR positive (58.8\%). The HER2

Table 1. Mean of quantitative variables in the study

\begin{tabular}{lc}
\hline variable & Mean \pm SD \\
\hline Ki67 & $16.85 \pm 16.63$ \\
age & $47.42 \pm 10.53$ \\
\hline
\end{tabular}

Table 2. Frequency and percent of qualitative variables in the study

\begin{tabular}{llc}
\hline Variable & & Frequency (percent) \\
\hline Ki67_cat & $<14$ & $203(55.6)$ \\
& $\leq 14$ & $162(44.4)$ \\
ER & - & $123(33.7)$ \\
& + & $242(66.3)$ \\
PR & - & $154(42.2)$ \\
& + & $211(58.8)$ \\
HER2 & - & $269(73.7)$ \\
P53 & + & $96(26.3)$ \\
& - & $199(54.5)$ \\
5 y DFS & + & $166(45.5)$ \\
& No & $162(44.4)$ \\
\hline
\end{tabular}

overexpression was identified in 96 tumors (26.3\%), other 269 patients had negative HER2 status (73.7\%). The P53 status in tumors was positive in 166 (45.5\%) and negative in 199 (54.5\%). Two hundred and three breast cancer patients had 5yDFS (55.6\%) compared with 162 patients (44.4\%) with no 5yDFS (Tables1 and 2).

Then the relation between 5yDFS and other characters were evaluated. We analyzed with univariate analysis. The $\mathrm{P}$-value $<0.2$ is considered significant.

The $67.5 \%$ and $40.7 \%$ of breast cancer patients respectively with low level of Ki67 and high level of Ki67 showed significant correlation with 5yDFS. The correlation between age and HER2 with $5 y D F S$ was not significant. We entered the variables with $\mathrm{P}$-value $<0.2$ in the multivariate analysis and calculated odds ratio which was 2.5 for ki67. The P-value $<0.05$ was considered significant (P-value $<0.001$ ). The chance of $5 \mathrm{yDFS}$ in breast cancer patients with low Ki67 in comparison with patients with high Ki67 was 2.53.

Odds ratio for correlation between ER and 5y DFS was 2.09 (P-value=0.068). This was not statistically significant but the level was important. Other correlations (PR, P53) with 5yDFS were not significant (Tables 3 and 4).

In another analysis, we evaluated correlation between Ki67 with other variables. In univariate analysis the correlation between ki67

Table 3. Univariate analysis of 5yDFs and related variables

\begin{tabular}{lllll}
\hline 5yDFS & no & Yes & P-value & Method \\
\hline $\begin{array}{llll}\text { Ki67 } \\
\text { Ki67_cat }\end{array}$ & $21.75 \pm 18.8$ & $12.94 \pm 13.48$ & $<0.001$ & Mann Whitney \\
$<14$ & $66(32.5)$ & $137(67.5)$ & $<0.001$ & Chi-square \\
$>14$ & $96(59.3)$ & $66(40.7)$ & & \\
age & $46.99 \pm 10.97$ & $47.77 \pm 10.17$ & 0.695 & Mann whitney \\
ER & & & & \\
- & $75(61)$ & $48(39)$ & $<0.001$ & Chi-square \\
+ & $87(36)$ & $155(64)$ & & \\
PR & & & & \\
- & $86(55.8)$ & $68(44.2)$ & $<0.001$ & Chi-square \\
+ & $76(36)$ & $135(64)$ & & \\
HER2 & $118(43.9)$ & $151(56.1)$ & 0.739 & Chi-square \\
- & $44(45.8)$ & $52(54.2)$ & & Chi-square \\
+ & $78(39.2)$ & $121(60.8)$ & 0.029 & \\
P53 & $84(50.6)$ & $82(41.4)$ & & \\
\hline
\end{tabular}


Table 4. Multivariate analysis of $5 y$ DFS and related variables

\begin{tabular}{lcccc}
\hline variable & B & SE & OR & P-value \\
\hline Ki67 & 0.926 & 0.229 & 2.53 & $<0.001$ \\
ER & 0.738 & 0.405 & 2.09 & 0.068 \\
PR & 0.103 & 0.385 & 1.11 & 0.79 \\
P53 & -0.192 & 0.229 & 0.83 & 0.4 \\
\hline
\end{tabular}

SE: standard error; B: unstandardized coefficient; OR: Odds ratio with ER, PR, HER2, and P53 was significant but this correlation was not significant for the age. Therefore, we entered these variables in multivariate analysis. The chance of high Ki67 in ER positive in comparison with ER negative was $0.3 / 1$ (P-value= 0.023). Odds ratio for HER2 was 0.5 indicating that the chance of high Ki67 in HER2 positive, in comparison with HER2 negative was 0.5/1 (Pvalue $=0.012$ ). Odds ratio for P53 was 2.204 (Tables $5,6)$.

Table 5. Univariate analysis of Ki67 and related variables

\begin{tabular}{lllll}
\hline Ki67 & $<14$ & $>14$ & P-value & method \\
\hline ER & & & & \\
- & $49(39.8)$ & $74(60.2)$ & $<0.001$ & Chi-square \\
+ & $154(63.6)$ & $85(36.14)$ & & \\
PR & & & & \\
- & $70(45.5)$ & $84(54.5)$ & 0.001 & Chi-square \\
+ & $133(63)$ & $78(37)$ & & \\
HER2 & & $127(47.2)$ & 0.069 & Chi-square \\
- & $142(52.8)$ & $35(36.5)$ & & \\
+ & $61(63.5)$ & $69(34.7)$ & $<0.001$ & Chi-square \\
P53 & $130(65.3)$ & $93(56)$ & & \\
- & $73(44)$ & $47.46 \pm 10.79$ & 0.656 & Mann Whitney \\
+ & $47.39 \pm 10.34$ & & & \\
age & & & & \\
\hline
\end{tabular}

Table 6. Multivariate analysis of Ki67 and related variables

\begin{tabular}{lllll}
\hline variable & B & SE & OR & P-value \\
\hline ER & -0.929 & 0.408 & 0.395 & 0.023 \\
PR & -0.089 & 0.393 & 0.915 & 0.822 \\
HER2 & -0.661 & 0.265 & 0.516 & 0.012 \\
P53 & 0.79 & 0.244 & 2.204 & $<0.001$ \\
\hline
\end{tabular}

B: unstandardized coefficient; OR : Odds ratio; SE: standard error

\section{CONCLUSION}

In this study we investigated the level of Ki67 and its relationship with 5y DFS. Previous studies have indicated the prognostic value of Ki67 in breast cancer. A number of studies stated that Ki67 in breast cancer patients with stage N0, N1 had prognostic value ${ }^{16}$. We did not find any association between age and 5y DFS. In one study reported that chance of relapse increased in breast cancer patients with high Ki67 (in lobular carcinoma in situ $)^{17}$. Our result was in invasive ductal carcinoma breast cancer patients that showed chance of 5yDFS increased in low Ki67 breast cancer.

In another study showed high Ki67 expression in post treatment tumors was strongly correlated with poor prognosis ${ }^{18}$. One of our findings was the increased level of 5yDFS in ER positive. The chance of high Ki67 is increased in the ER negative, HER2 negative, and P53 positive breast cancer patients.

\section{REFERENCES}

1. Torre, L.A., et al., Global cancer statistics, 2012. CA: a cancer journal for clinicians, 2015; 65(2): p. 87-108.

2. Siegel, R.L., K.D. Miller, and A. Jemal, Cancer statistics, 2015. CA: a cancer journal for clinicians, 2015; 65(1): p. 5-29. 
3. Mousavi, S.M., et al., Cancer incidence and mortality in Iran. Annals of Oncology, 2009; 20(3): p. 556-563.

4. Leong, S.P., et al., Is breast cancer the same disease in Asian and Western countries? World journal of surgery, 2010; 34(10): p. 2308-2324.

5. Chia, S.K., et al., The impact of new chemotherapeutic and hormone agents on survival in a population based cohort of women with metastatic breast cancer. Cancer, 2007; 110(5): p. 973-979.

6. Gennari, A., et al., Survival of metastatic breast carcinoma patients over a 20 year period. Cancer, 2005; 104(8): p. 1742-1750.

7. Dafni, U., et al., Fifteen-year trends in metastatic breast cancer survival in Greece. Breast cancer research and treatment, 2010; 119(3): p. 621631.

8. De Azambuja, E., et al., Ki-67 as prognostic marker in early breast cancer: a meta-analysis of published studies involving 12155 patients. British journal of cancer, 2007; 96(10): p. 15041513.

9. Hanahan, D. and R.A. Weinberg, The hallmarks of cancer. cell, 2000; 100(1): p. 57-70.

10. Di Lorenzo, G., et al., Expression of epidermal growth factor receptor correlates with disease relapse and progression to androgenindependence in human prostate cancer. Clinical Cancer Research, 2002; 8(11): p. 3438-3444.

11. Yadollahpour, A. and Z. Rezaee, Electroporation as a New Cancer Treatment Technique: A Review on the Mechanisms of Action. Biomedical \& Pharmacology Journal, 2014; 7(1): p. 53-62.

12. Arap, W., R. Pasqualini, and E. Ruoslahti, Cancer treatment by targeted drug delivery to tumor vasculature in a mouse model. Science, 1998; 279(5349): p. 377-380.

13. Ali, Y. and S. Hamed, Early Breast Cancer Detection using Mammogram Images: A Review of Image Processing Techniques. Biosci., Biotech. Res. Asia, 2015; 12(Spl.Edn.1): p. 225-234.

14. Urruticoechea, A., I.E. Smith, and M. Dowsett, Proliferation marker Ki-67 in early breast cancer. Journal of clinical oncology, 2005; 23(28): p. 7212-7220.

15. Scholzen, T. and J. Gerdes, The Ki-67 protein: from the known and the unknown. Journal of cellular physiology, 2000; 182(3): p. 311-322.

16. Strand, C., et al., The combination of Ki67, histological grade and estrogen receptor status identifies a low-risk group among 1,854 chemonaïve women with N0/N1 primary breast cancer. SpringerPlus, 2013. 2(1): p. 111.

17. Vincent-Salomon, A., et al., High Ki67 expression is a risk marker of invasive relapse for classical lobular carcinoma in situ patients. The Breast, 2012; 21(3): p. 380-383.

18. Tan, Q.-X., et al., Prognostic value of Ki67 expression in HR-negative breast cancer before and after neoadjuvant chemotherapy. International journal of clinical and experimental pathology, 2014. 7(10): p. 6862. 